\section{Folia Phoniatrica et Logopaedica}

\title{
Revised IALP Education Guidelines (September 1, 2009): IALP Guidelines for Initial Education in Speech-Language Pathology ${ }^{1}$
}

\section{Preamble}

In 1995, the IALP (International Association of Logopedics and Phoniatrics) Board approved the Guidelines for the Initial Education in Logopedics. The guidelines were published in Folia Phoniatrica et Logopaedica in 1995. They have been used extensively to support and inform those interested in the development of new education courses/programs and the revision of existing course curricula/programs around the world.

Since the original adoption, many cultural, political and educational changes have taken place globally. Several countries are developing an awareness of the need to provide services to persons with disabilities. Others are finding it necessary to expand their education programs to meet the needs of an expanding client base. It was necessary to review the educational guidelines for their current appropriateness.

Members of the Logopedics Education Committee met at the 2007 IALP Congress in Copenhagen, Denmark, and again in Ljubljana, Slovenia, in 2009 and agreed to contribute to the review of the education guidelines and revise as necessary. The following is the revised version.

It should be noted that these guidelines refer only to the education of speech-language pathologists (or their equivalent in other terminologies) as defined in the Appendix. The IALP recognizes that some countries may choose to train people whose work includes helping people with communication disorders via alternative or additional routes than by setting up academic programs of the type illustrated here. The guidelines have been devised with the intention of describing patterns of good practice in the education of professionals identified as speech-language pathologists, which many programs at present follow, and which other programs may wish to bear in mind in seeking to work towards an international framework.

The IALP Education Committee recognizes that a variety of social, cultural and educational influences need to be taken into consideration in planning programs in different countries, and that this may be particularly important where new programs are initiated for the first time in a country. Following the tremendous changes that have taken place globally during the last decades, it is appropriate to describe different educational routes because the profession developed at different levels in dif-

\footnotetext{
I In the original guidelines, the term 'logopedics' was used to identify the professional area. Although the term is used in many places throughout the world, currently the title most commonly used in the USA and Canada is 'speech-language pathology'. 'Speech-language therapy' is used in most English-speaking countries. Throughout this revision, the term 'speechlanguage pathology' will be used to identify the professional area. Persons using these guidelines are free to use the title as used in their country or locale. It is recommended that terms such as 'generic' or 'discipline-related competencies', 'master's level' and 'bachelor's level' be adopted and defined for the specific country.
}

\section{KARGER}

Fax +41 613061234

E-Mail karger@karger.ch

www.karger.com
(C) 2010 S. Karger AG, Basel

$1021-7762 / 10 / 0625-0210 \$ 26.00 / 0$

Accessible online at: www.karger.com/fpl 
ferent parts of the world. In some countries, speech-language pathology education programs are well established. In other countries, such programs are in the development stage. In still other countries, the programs may be just beginning or may be in the planning stages. These guidelines relate to each of the three levels of programs. The guidelines are not intended to substitute for the accreditation requirements set by national professional bodies. For the countries without specific accreditation and evaluation of requirements in speech pathology, these guidelines can serve as a reference for the establishment of a national standard.

While it is recognized that the education of speechlanguage pathologists is a lifelong pursuit, these guidelines relate only to the initial education of speech-language pathologists, rather than to their continuing professional development following qualification.

\section{Part A: Premises}

\section{Background}

The purposes of providing guidelines for the education of speech-language pathologists are to harmonize the initial education of speech-language pathologists, to develop and maintain appropriately high standards of education and, in due course, to facilitate the international movement of personnel and knowledge. It is hoped that through the dissemination of these guidelines to all educational programs in speech-language pathology, competent and innovative practitioners will be able to do the following:

(a) offer and/or improve quality service to people with communication and/or swallowing disorders in countries where initial educational programs are already established;

(b) improve the service to people with communication and/or swallowing disorders in countries where educational programs are developing;

(c) establish new programs for the initial professional education of speech-language pathologists in countries where such programs do not currently exist.

Surveys of the education of speech pathologists worldwide have indicated that:

(a) a variety of approaches to the initial education of speech-language pathologists currently exist;

(b) in some countries, education in speech-language pathology is undertaken simultaneously with education in a second profession, i.e. audiology/hearing therapy, education, psychology or general rehabilitation.
The Statement of Premises and the Illustrative Framework below provide a general statement of principles to be taken into consideration in the education of speechlanguage pathologists, with allowance for the practices of different cultures and in different countries. Where it is the practice to combine education in speech-language pathology with that in another profession, these guidelines relate specifically and strongly to the speech-language pathology component of the education. This is to ensure that this discipline should be sufficient to stand on its own and be comparable to such programs in which it is an independent field.

\section{Statement of Premises}

(1) Speech-language pathology is an identifiable, independent profession in its own right, and is not one whose practitioners are seen as educational/medical/ social assistants.

(2) Practitioners follow a code of ethics, which specifies the level of training and responsibilities, the recognition of the boundaries of their work and skills, and the need to consult with and refer clients to other professionals, as appropriate.

(3) The education of speech-language pathologists is aimed at preparing professionals for a broad and general scope of work with people of all ages who may have communication and/or swallowing disorders.

(4) The education of speech-language pathologists includes an appreciation of cultural and linguistic factors that influence the development of speech and language and the delivery of appropriate clinical services.

(5) The education of professional practitioners includes advanced knowledge about the nature of communication disorders and the provision of culturally appropriate diagnosis and intervention.

(6) The education of professionals includes an appreciation of evidence-based practice and the use of research to support clinical approaches.

(7)Speech-language pathologists should be given opportunities to develop their skills under supervision for their first year of experience in the profession.

(8) Opportunities should be provided for speech-language pathologists to continue their professional development through continuing education, specialization and studying for advanced degrees which foster research in communication disorders. 


\section{Part B: Illustrative Framework}

\section{Principles}

A fundamental principle of education in speech-language pathology is the recognition of the complexity of the field of human communication and its development, and of communication impairments and disabilities.

1.1 The program should make students aware of the complexity of human communication and its disorders as well as of normal/disordered swallowing.

1.2 The study of communication disabilities should be based on the foundation of a study of normal communication and its development.

1.3 The program should integrate the teaching of theory with the teaching of the practical applications of theory, and include a substantial element of clinical practicum to achieve clinical competence as generic therapists.

1.4 The program should include an awareness of social, linguistic and cultural differences both within and across countries, and a respect for differences both amongst individuals and amongst societies. Students should be made aware of multilingual issues within specific communities. They should be informed about the possible role of the speech-language pathologist in establishing interprofessional networks in their communities.

\section{Content}

2.1 The study of speech-language pathology is highly related to the disciplines of psychology and linguistic sciences, behavioral sciences, biomedical sciences and ethical issues. The program should cover the main concepts of the supporting disciplines. Such coverage should provide the students with an overview of the relevance of the main concepts of each discipline, and detailed study of such theories and approaches that are directly relevant to the understanding of human communication and its disorders.

\section{Supporting Disciplines}

(a) Linguistic Sciences

Linguistics, language acquisition, sociolinguistics, multilingualism, phonetics and acoustics are relevant to linguistic sciences. The study should include speech sounds, phonology, syntax, semantics, lexicons, discourse and pragmatics, with practical work in data collection, transcription, measurement and analysis (including qualitative analysis of oral as well as written language). It should also include discourse analysis, es- pecially in relation to disordered speech and language. The content of the above-mentioned domains of study should be relative to the language of the country.

(b) Behavioral Sciences

Studies in behavioral sciences should include cognitive psychology, social psychology, developmental psychology (across the life span), neuropsychology, education/pedagogy and studies of personality and individual differences, with guided fieldwork, particularly in relation to understanding psychological assessment.

\section{(c) Biomedical Sciences}

Studies in biomedical sciences should include biological bases of language, speech and swallowing (human anatomy and physiology as well as neuroanatomy), and clinical medical sciences as applied in neurology, otorhinolaryngology, pediatrics, geriatric medicine, psychiatry (across the age span), audiology, orthodontics and the study of craniofacial anomalies and their repair and of deglutition. Opportunities for observing clinical (especially multidisciplinary) sessions in these related disciplines should be included.

The teaching in the above-mentioned supporting disciplines should preferably be provided by qualified specialists in each field who are sensitive to the specific relevance of their field to the needs of speech-language pathologists.

(d) Ethical Issues

Students should have knowledge of relevant ethical guidelines for research and practice. They should be familiar with laws, statutes and regulations concerning professionals in private and public social and health care organizations. Throughout the entire program, students should follow these ethical principles in their theoretical and practical studies. The program should use professionally and scientifically qualified teachers. Teachers providing clinical supervision need to hold the professional certificate in speech-language pathology required in the local country.

2.2 In addition to the above supporting sciences, the program should cover the principle areas of the discipline. This should include the study of the following:

(a) the varieties of normal and abnormal communication, their characteristics and possible causal factors/etiologies as well as interpretations of their nature from biological, cognitive and sociocultural perspectives;

(b) theories of the assisted establishment/recovery of language function; 
(c) culturally and linguistically appropriate methods and resources for assessment and diagnosis;

(d) methods of evaluating the effectiveness of diagnosis and intervention;

(e) the consequences of communication disorders for the families and social contacts and methods of counseling;

(f) the social and organizational settings in which speech-language pathologists work with respect to health, education, the work of allied professionals, legal and ethical issues, the use of resources, and professional responsibility;

(g) the teaching of logopedics should be provided by qualified speech-language pathologists who maintain an active involvement in clinical work and have clinical research experience in specific areas of speech-language pathology.

2.2.1 The study of logopedics must include practical work carried out under the supervision of qualified and experienced speech-language pathologists and monitored by the educational program. This should be aimed at enabling the student to acquire general skills and systematic methods of working with clients. It should foster the personal development of the student and interactive communication skills. It is necessary that the supervisors have current knowledge of the profession and be trained in supervision.

2.2.2 The practicum should show how the studies identified under 2.2, letters a-g, are applied. It is, therefore, recommended that the practicum is undertaken in association with the teaching of the theory components outlined in 2.2, in order to facilitate the integration of theory and practice.

2.2.3 The practicum should begin with the observation of skilled practitioners and continue with direct interactive experience for each student in a variety of settings, with a variety of types of clients, and with a variety of responsibilities from screening to diagnosis, and from planning to applying intervention programs.

2.2.4 Students should receive training with a variety of cases. Direct experience in a clinical practicum should include work with at least the following types of disorders:

(a) Developmental and acquired speech disorders of a phonetic and/or phonological nature in children and disorders which predominantly or also involve other levels of language organization in both children and adults

(b) Voice and resonance disorders

(c) Fluency disorders

(d) Swallowing disorders

(e) Reading and writing disorders
2.2.5 In addition, the program should provide some practical experience for the student of cases with communication or oral disorders secondary to at least some of the following:

(a) Hearing impairment

(b) Cognitive impairment and disability

(c) Language learning impairment and disability

(d) Behavioral and emotional disabilities (e.g. autism, attention deficit)

(e) Psychiatric disabilities (e.g. schizophrenia, psychosis, the dementias)

(f) Structural abnormalities including congenital (e.g. cleft palate) and acquired abnormalities (e.g. laryngectomy)

(g) Cerebral palsy and other neuromotor impairment

(h) Swallowing and feeding disabilities (see 2.2.4 above)

(i) Symptoms secondary to social deprivation

(j) Multiple and complex impairments and disabilities (e.g. combinations of any of the above)

To supplement (but not substitute for) the direct practical experience of some of the above, video-taped recordings and web-based technology (preferably interactive) may be used in order to make students aware of work with the other categories of disabilities if direct access to such client groups is not possible.

2.2.6 There should be a practical examination of the student's clinical work at or near the end of the program, in which the student's ability to apply theory to practice is assessed. It is also recommended that there should be frequent in-course assessment of students during the program to allow opportunity for remedial help or redirection, if necessary.

2.2.7 Clinical guidelines for supervisors should be provided.

\section{Structure}

\subsection{For Countries where the Service to People with} Communication Disorders Is Already Well Established

In order to achieve the requisite competencies related to the profession of speech-language pathology, the educational program giving access to the profession must be undertaken at the university or equivalent academic level. If possible, a doctoral degree and other forms of specialization should be offered in accordance with the traditions of the organizing university, academic institution or specialized scientific committee. 
The educational program should be in balance with the generic competencies related to an equivalent academic degree and in accordance with discipline-related competencies described as an integration of knowledge, understanding, discipline-specific skills and abilities, and should be organized in three competency areas:

(a) clinical practice - competency in prevention, assessment, diagnostics, training and therapy related to clients and their community;

(b) organization and administration - understanding the requirements for working in and for an organization in the country including an understanding of the laws;

(c) professionalization - understanding the principles of professional conduct, and expectations of the development of the profession and the discipline.

\subsection{For Countries where the Service to People with}

Communication Disorders Is Developing

Two educational routes providing access to the profession are considered acceptable:

(a) first-degree generic competencies obtained at the bachelor or equivalent level, preferably distributed over 4 years and covering all necessary domain-specific competencies related to good practice of the profession;

(b) a professional postgraduate or equivalent degree in speech-language pathology, following a first-degree course in logopedics of at least 3 years; it is recommended that the postgraduate program be distributed over at least 2 years.

\subsection{For Countries where the Professional Education} of Speech-Language Pathologists Does Not

Currently Exist and where the Service to People with

Communication Disorders Is Not Yet Established

The purpose of this section is to facilitate the initiation of appropriate professional education in speech-language pathology in countries that have chosen to establish new programs for the education of practitioners in a speechlanguage pathology service. It is recognized that some countries may choose initially to develop other patterns of service which include help for persons with communication disorder, and that different means of training community workers might be used as a supplement to, or instead of, education in speech-language pathology. The prime motivation for such education should arise indigenously, with consideration of the resources of the country and the usual pattern of the delivery of health care and education in the community.

A crucial first stage should be to identify major cultural issues of providing the service in the community and to evaluate the needs of people in the community. This should include a review of existing services, resources and barriers to the establishment of the service within the prevailing local context. The local education system should be taken into consideration when developing and planning a new program.

In such cases, the program should begin with external advisers acting as facilitators rather than directors. External facilitators should be sensitive to aspects of indigenous culture and circumstances, and some points for consideration are given below:

(a) sustainability, financing and the intermeshing of the speech-language pathology service with other existing health and educational services;

(b) materials such as textbooks and other resources must be evaluated for appropriateness for the culture and circumstances, particularly in respect of the pictorial illustrations included;

(c) the stated cultural value or purpose of rehabilitation in the country must be considered, and the program must be culturally appropriate;

(d) the equipment and technical resources need to be appropriate to the circumstances, and the availability of technical support staff is essential;

(e) where other professional services are unavailable, workers treating individuals with communication disorders are likely to extend the boundaries of their professional training beyond that which is conventionally acceptable in better resourced countries and, therefore, workers and organizers need to be made aware of the points at which such tolerance may endanger the welfare of the persons served;

(f) in countries where a speech-language pathology service does not exist, a local clinical practicum for students may be impossible, and the appropriateness of placing these students in well-resourced countries for their clinical experience needs to be carefully considered; alternatively, it may be suggested that experienced professionals are brought in to build this practicum;

(g) a greater emphasis in initial training may need to be laid on the management role of communication workers and their role in training the skills of others; students should be trained to provide speech-language pathology services within the construct of health care services and educational services in the country;

(h) there may be linguistic variations and a range of coexisting languages which will have implications for the educational program;

(i) the program content may need to be adjusted to facilitate meeting the local needs; 
(j) students should be made aware of the limitations of using materials and resources which have been developed by other countries (or in other regions of the same country) for different cultural and linguistic needs;

(k) steps should also be taken to initiate the development of an appropriate status for the profession and career progression of its practitioners.

\section{Research}

Academics on the programs should be active in research in speech-language pathology and/or its supporting disciplines, so as to stimulate interest in research and to keep academics and students up to date with current developments in these fields, e.g. with single-case studies of behavioral analysis in clinical assessment.

All programs should include a research project to foster a research-oriented (evidence-based) approach to clinical work, and to assist the student in the critical examination of research in the field.

\section{Program Evaluation}

Programs should periodically undertake self-evaluations to determine whether they are satisfactory, whether the guidelines have been adequately met, and how the program can be improved.

\section{Continuing Education and Scientific Study}

Speech-language pathologists should continue to maintain their competence by updating their knowledge and skills. They should contribute to the development of the discipline and of the profession by undertaking and publishing research. Experienced speech-language pathologists have a responsibility to assist and tutor students of their profession and supervise their clinical practice.

\section{Appendix}

Definition and Roles of the Speech-Language Pathologist

The definition and roles of the speech-language pathologists have developed and expanded during the 20th century. Scientific and technical developments, along with changes in law and funding relating to the provision of health and education services, will influence the definition and roles of the speech-language pathologist.

Definition

The central concern of the profession of speech-language pathology is that people with communication and swallowing disabilities (dysphagia) receive the best possible service to alleviate their disabilities and improve their quality of life. To achieve these goals, the speech-language pathologist is involved in the prevention, assessment, intervention, management and scientific study of disorders of human communication and of swallowing. In this context, human communication comprises all those processes and functions associated with the production of speech, and with the comprehension and production of oral and written language, as well as forms of nonvocal communication. Swallowing refers to safe transit of food and drinks through the oropharynx to ensure optimal oral nutrition.

Roles and Functions of the Speech-Language Pathologist

Speech-language pathologists require both scientific knowledge and clinical competence in order to provide optimal levels of client care. The speech-language pathologist has the roles stated in the following.

Prevention

The speech-language pathologist is concerned with the prevention of the occurrence or development of communication disorders by:

(a) education of the public and other professionals about the nature of communication and the prevention of communication disorders;

(b) early identification of communication disorders and factors directly associated with communication disorders;

(c) collaboration with other professionals as relevant to the role of the speech-language pathologist in the prevention of communication disorders.

\section{Assessment}

Assessment is a continuing process, and in most cases, instances will involve collaboration with other disciplines. A diagnosis is reached via objective testing and observation, and consultations with the client/family members and other professionals as necessary. This leads to a hypothesis about the nature and duration of a targeted intervention.

Intervention

The speech-language pathologist carries out interventions in communication disorders and dysphagia to assist clients to achieve the best possible function and to reduce or eliminate the impact of the primary impairment. Intervention represents a joint undertaking between the speech-language pathologist and the client/family and subsumes client management including the selection of goals and therapy procedures. Intervention goals are 
based on assessment and client/family priorities and may include early intervention, rehabilitation, counseling, consultation and participation in management by teamwork. An essential part of intervention is the evaluation of its efficacy.

\section{Professional Conduct}

Speech-language pathologists must maintain professional responsibility for the welfare of their clients at all times. They must observe the code of ethics of their national professional body, and/ or as prescribed by their employer and/or their national/state government.
Continuing Education and Scientific Study

It is an ethical responsibility of speech-language pathologists to participate in continuing education and scientific study to update their knowledge and skills, and to maintain their competence to practice. Where possible, speech-language pathologists should contribute to the development of the discipline and of the profession by undertaking and publishing research and therapy reports. Experienced speech-language pathologists have a responsibility to assist and tutor students of their profession and supervise their clinical practice. 